\title{
Market Sentiment: A Tragedy of the Commons
}

\author{
By Tarek A. Hassan and Thomas M. Mertens*
}

A prominent idea in behavioral finance is that individuals have some propensity to make correlated mistakes in their investment decisions. The leading critique of this idea is that investors who make systematic mistakes suffer a utility cost and should be expected to alter their behavior. In this paper we present a noisy rational expectations model in which investors decide whether or to what degree they want to allow their behavior to be influenced by "market sentiment", which we define as a bias in individual beliefs about future payoffs. Investors who choose to insulate their decision from market sentiment earn higher expected returns, but incur a small mental cost. (It requires some concentration to insulate oneself from the moods of others.)

We show that if information is moderately dispersed across investors, even a very small mental cost (on the order of $0.001 \%$ of consumption) may generate a significant amount of sentiment in equilibrium: Individuals who choose to be swayed by sentiment increase uncertainty about the future and make it less costly for others to be swayed by sentiment as well. Market sentiment thus emerges as a tragedy of the commons. In related work, (Tarek A. Hassan and Thomas M. Mertens 2010), we show that even moderate amounts of this kind of sentiment may result in a large rise in the financial risk faced by investors.

\section{Setup}

There is a continuum of agents on the interval $[0,1]$. Each agent $i$ allocates his initial wealth $w_{0 i}$ between a riskless and a risky asset. For each unit purchased the

* Hassan: University of Chicago, Booth School of Business, 5807 S Woodlawn Ave., Chicago IL 60637, USA, tarek.hassan@chicagobooth.edu. Mertens: New York University, Stern School of Business, $44 \mathrm{~W}$ Fourth St., Suite9-73, New York NY 10012, USA, mertens@stern.nyu.edu. riskless asset yields one unit, the risky asset $X$ units of a single consumption good, where $X$ is normally distributed with mean $\bar{X}$ and variance $\sigma_{X}^{2}$. The portfolio of an agent holding $z_{i}$ units of the risky asset returns $w_{1 i}=w_{0 i}+z_{i}(X-p)$, where $p$ is the period 0 price of the risky asset.

Period 0 consists of two sub-periods: At the end of period 0 , agents observe $p$ and receive a private signal about the payout of the risky asset, $s_{i}=X+\nu_{i}$, which contains idiosyncratic noise $\nu_{i} \sim N\left(0, \sigma_{\nu}^{2}\right)$. Given these two pieces of information, agents choose $z_{i}$ to maximize the conditional expectation of their utility.

At the beginning of period 0 , and before receiving any information about $X$, each agent chooses whether or to what degree $\mu_{i}$ he will allow market sentiment $\varepsilon$ to influence his portfolio decision at the end of period 0 . An agent who allows market sentiment to sway his decision has his posterior distribution shifted by $\mu_{i} \varepsilon$ and thus holds a distorted conditional expectation, $\hat{E} X+\mu_{i} \varepsilon$, where $\hat{E} X$ is agent $i$ 's rational expectation of $X$ given $s_{i}$ and $p$. Agents who choose not to insulate their decisions from market sentiment are thus slightly too optimistic about $X$ when sentiment is positive and slightly too pessimistic when sentiment is negative. Market sentiment, $\varepsilon$, is normally distributed with mean 0 . The variance of market sentiment, $V^{\varepsilon}$, is an endogenous variable for which we solve below. Taking $V^{\varepsilon}$ as given, agents choose $\mu_{i}$ to maximize the unconditional expectation of their utility, $E U_{i}$, where $E$ is the unconditional expectations operator and the utility function $U_{i}$ exhibits constant absolute risk aversion with

$$
U_{i}=-\frac{1}{\rho} e^{-\rho w_{1 i}\left(1-\kappa\left(1-\mu_{i}\right)\right)} ;
$$

where $\rho$ is the coefficient of absolute risk aversion, and $\kappa$ represents a small mental cost incurred by agents who choose to insu- 
late their decisions from market sentiment. In particular, an agent who chooses to eliminate market sentiment from his decision incurs a mental cost equivalent to $100 \cdot \kappa \%$ of consumption. Agents thus face a trade off between making a small mistake $\mu_{i} \varepsilon$ in their portfolio allocation and incurring the small mental cost $\kappa\left(1-\mu_{i}\right) w_{1 i}$.

An equilibrium of this economy is a combination of $p, V^{\varepsilon},\left\{\mu_{i}\right\}$, and $\left\{z_{i}\right\}$ at which agents maximize their utility, markets clear, $Z=\int z_{i} d i$, and no agent chooses a higher or lower exposure to market sentiment than any other agent, $\mu_{i}=1 \forall i$; where we consider only the symmetric linear equilibrium.

\section{Equilibrium Sentiment}

We solve the model in two steps. We first calculate agents' optimal portfolio choice and the equilibrium price, taking as given $\mu_{i}$ and $V^{\varepsilon}$. We then calculate $E U_{i}$, the optimal choice of $\mu_{i}$, and equilibrium $V^{\varepsilon}$.

The first step of the solution is similar to solving a standard noisy rational expectations model: We guess that the equilibrium price takes the linear form

$$
p=\pi_{0}+\pi_{1} X+\gamma \varepsilon .
$$

Given this linear price function the conditional expectation of $X$ takes the form $\hat{E} X=\alpha_{0}+\alpha_{1} y_{i}+\alpha_{2} p$. Plugging (2) into this expression and matching coefficients yields

$$
\pi_{1}=\frac{\alpha_{1}}{1-\alpha_{2}}, \text { and } \gamma=\frac{1}{1-\alpha_{2}} .
$$

We then use this result in combination with the projection theorem to solve for the constants $\alpha_{0}, \alpha_{1}, \alpha_{2}$, and $\pi_{0}$. Given these constants, we can solve for the equilibrium price $p$, the conditional expectation of utility, $\hat{E} U_{i}$, as well as the conditional and unconditional variances of returns, $\hat{V}^{X-p}$ and $V^{X-p}$, respectively.

The crucial step in the solution is to solve for the unconditional expectation of agents' utility. As agents are mean variance optimizers, their terminal wealth is a function of the product of two normally distributed variables, $z_{i}$ and $X-p$. We obtain a closed form solution for $E U_{i}$ by re-writing $\hat{E} U_{i}$ as a function of two mean-zero distributed variables $\left(\hat{E} X-p-(\bar{X}-\bar{p})\right.$ and $\mu_{i} \varepsilon$, where $\left.\bar{p}=\pi_{0}+\pi_{1} \bar{X}\right)$ and then using the formula for the unconditional expectation of a Wishart variable given in (Markus K. Brunnermeier 2001, p. 64). We get

$$
E U_{i}=-\frac{e^{-w_{0} \rho\left(1-\kappa\left(1-\mu_{i}\right)\right)-\frac{(\bar{X}-\bar{p})^{2}\left(1-\mu_{i}^{2} V^{\varepsilon} \phi_{1}\right)}{2\left(V^{X}-p-\mu_{i}^{2} V^{\varepsilon}\left(1+\phi_{2}\right)\right)}}}{\rho \sqrt{\frac{V^{X-p}-\mu_{i}^{2} V^{\varepsilon}\left(1+\phi_{2}\right)}{\hat{V}^{X-p}}}},
$$

where $\phi_{1}=\frac{\alpha_{1}^{2} \sigma_{\nu}^{2}}{\hat{V}^{X-p}}$ and $\phi_{2}=\frac{1}{\hat{V}^{X-p}} \cdot 1$

This expression has an intuitive interpretation: If the agent chooses $\mu_{i}=0$, utility rises with the unconditional Sharpe Ratio (the second term in the exponent reduces to $\left.\frac{\bar{X}-\bar{p}}{V^{X-p}}\right)$, and with the ratio of the unconditional to the conditional variance of returns (the term in the denominator reduces to $\left.\frac{V^{X-p}}{\hat{V}^{X-p}}\right)$. We may think of this latter ratio as the extent to which the agent has an informational advantage over the market. For $\mu_{i}>0$, both ratios are slightly lower. Sentiment thus lowers the agent's unconditional Sharpe Ratio and destroys part of his informational advantage.

However, it is also apparent from (4) that agents will always allow some influence of sentiment on their decisions as the utility cost of sentiment to the individual is proportional to $\mu_{i}^{2}$, while the mental cost of avoiding sentiment is proportional to $\mu_{i}$. Moreover, the utility cost of sentiment remains small even for $\mu_{i}=1$ as long as $V^{\varepsilon}$ is small relative to $V^{X-p}$ and $\hat{V}^{X-p}$. In fact, the effect of $V^{\varepsilon}$ on the utility cost of sentiment is in each case proportional to either $\phi_{1}$ or to $\phi_{2}$, which are both inversely related to the conditional variance of returns. One can show that both of these factors monotonically go to zero as $\sigma_{\nu} \rightarrow \infty$. The more dispersed information is in the economy the smaller is thus the utility cost of a given amount of sentiment.

We may now take the first order condition

\footnotetext{
${ }^{1}$ For the solution of similar models see (Hassan and Mertens 2010, section 3.2) and (Stijn Van Niewerburgh and Laura Veldkamp 2009, section 2.1).
} 
of (4) with respect to $\mu_{i}$, plug in $\mu_{i}=1$ and solve for the equilibrium $V^{\varepsilon}$. As the analytical solution for $V^{\varepsilon}$ is somewhat unwieldy we consider a numerical example in which $\rho=5$ to gain intuition for the results. To facilitate the interpretation of the results, we scale all standard deviations with the amount of real risk in the economy, $\sigma_{X}$. With this scaling all standard deviations have a natural interpretation. In particular, the ratio $\left(\frac{\sigma_{\nu}}{\sigma_{X}}\right)^{2}$ measures the level of dispersion of information in the economy as the number of of individuals who, in the absence of a market price, would need to pool their private information to reduce the conditional variance of $X$ by one half.

Panel A of Figure 1 plots the equilibrium standard deviation of sentiment as a percentage of the real risk in the economy, $\frac{\sqrt{V^{\varepsilon}}}{\sigma_{X}} \cdot 100$, over the mental cost of eliminating all sentiment from one's investment decision as a percentage of consumption, $\kappa \cdot 100$. The solid black line plots the results for $\frac{\sigma_{\nu}}{\sigma_{X}}=10$. The most striking result from the plot is that even what we might consider to be economically infinitessimal mental costs are sufficient to generate a significant amount of sentiment in equilibrium. For example, a cost of $0.003 \%$ of consumption is sufficient to generate a standard deviation of sentiment of approximately $1 \%$ of the amount of real risk in the economy. There are two, mutually reinforcing, mechanisms underlying this finding.

The first is of course the envelope theorem: From the perspective of the individual agent, making a marginal mistake around the optimal portfolio decision is cheap and therefore only larger amounts of sentiment affect the agent's utility at all (hence the monotonic but concave shape of the function). The second is a general equilibrium effect: The higher is the equilibrium amount of sentiment in the economy the higher is $\hat{V}^{X-p}$, which lowers the utility cost of sentiment to the individual. In other words, more sentiment increases uncertainty about the future, which makes it harder for others to hedge against sentiment, and thus lowers the cost to the individual agent of allowing it to affect his decisions. A small mental cost to the individual investor thus generates an equilibrium amount of sentiment which is several orders of magnitude larger.

The latter mechanism is attributable to an externality which we study in (Hassan and Mertens 2010): In settings in which asset prices aggregate dispersed information, smart investors should use prices to learn about the future. But if investors learn from prices, anything that moves equilibrium prices affects the equilibrium expectations of all market participants. Small correlated errors in investor behavior, such as the market sentiment in the present model, may thus lead to much larger changes in the equilibrium price. This is apparent from equation (2), where $\varepsilon$ is multiplied with $\gamma$, which from (3) is larger than one. Even a small $V^{\varepsilon}$ may thus lead to a large amount of noise in the equilibrium price and, consequently, to a reduction of its information content. When the price contains less information, investors face more uncertainty about the future and the conditional and unconditional variance of equilibrium returns rises. A small $V^{\varepsilon}$ may thus generate large amounts of financial risk. This is apparent in Panel B of Figure 1 , which plots the standard deviation of returns as a percentage of the real risk in the economy, $\frac{\sqrt{V^{X-p}}}{\sigma_{X}} \cdot 100$, over the mental cost of avoiding sentiment. The solid black line again plots the results for $\frac{\sigma_{\nu}}{\sigma_{X}}=10$. When $\kappa=0$ agents face no financial risk at all, as the equilibrium price perfectly reveals $X$ in the absence of any interference, but as soon as $\kappa>0$, the amount of financial risk rises dramatically. At $\kappa=0.00003$, the standard deviation of returns is $80 \%$ of the standard deviation of $X$ and thus rapidly approaches its upper bound.

The dashed and dotted lines in Figure 1 give a comparative static for varying levels of dispersion of information ranging from $\frac{\sigma_{\nu}}{\sigma_{X}}=1$ (one private signal reduces the conditional variance by half) to $\frac{\sigma_{\nu}}{\sigma_{X}}=40$ (1600 private signals reduce the conditional variance by half). In Panel $\mathrm{A}$, a given $\kappa$ generates a larger $V^{\varepsilon}$ when information is more dispersed, as more dispersed information results in a higher conditional variance and thus lowers the utility cost of sentiment to an individual agent. In Panel B, this comparative static is reinforced by another ef- 
Panel A

$$
\sqrt{V^{\epsilon}} / \sigma_{X} \cdot 100
$$

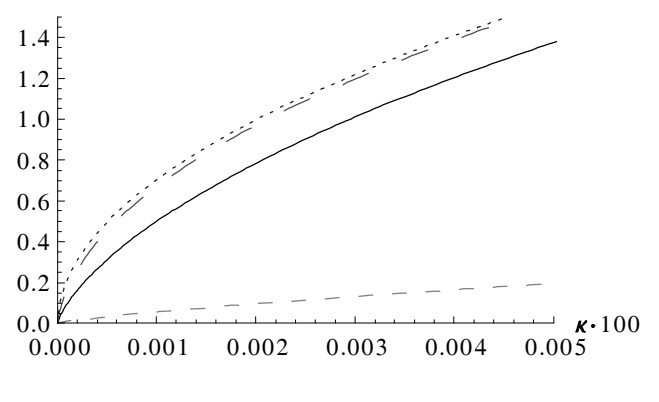

$\sigma_{v} / \sigma_{X}=1 \quad \sigma_{v} / \sigma_{X}=10$
$\sqrt{V^{X-p}} / \sigma_{X} \cdot 100$

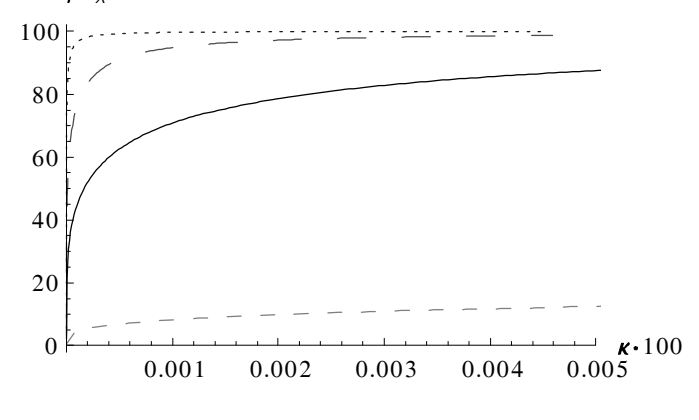

Panel B

Figure 1. Equilibrium sentiment (Panel A) and equilibrium variance of Returns (Panel B) plotted over mental cost of eliminating all Sentiment.

fect which we have documented in our earlier work: that a given $V^{\varepsilon}$ has a more detrimental effect on information aggregation when information is more dispersed. When information is more dispersed, a given $\kappa$ thus generates more sentiment and a given amount of sentiment simultaneously generates more financial risk. The cumulation of these two effects leads to the striking conclusion that even a mental cost on the order of $0.001 \%$ of consumption may result in an amount of equilibrium sentiment which is sufficient to completely eliminate all information from the market price.

\section{Discussion}

This striking link between small mental costs and significant market sentiment is more general than the simple environment we presented here. For example, the same logic holds if there is aggregate noise in the private signal (such that the sum of individual signals does not add up to $X$ ), or if individuals observe a noisy public signal in addition to their private signal. Both additions to the model merely change how much information is available to be aggregated by financial markets. The mental cost of avoiding market sentiment on the other hand determines how well this information is aggregated. The mechanism we described in this paper is thus relevant in any setting in which financial markets are considered to play an important role in aggregating information.

This paper is part of a broader research agenda in which we explore the role of individual incentives for information aggregation and the functioning of financial markets. In (Hassan and Mertens 2010) we solve a real business cycle model in which information is dispersed and households make small, correlated errors in their investment behavior. (Thomas Mertens 2009) explores policy implications and develops a general solution method for non-linear models with dispersed information. In (Tarek A. Hassan and Thomas M. Mertens 2011) we explore the trade-off between information acquisition and precision in the implementation of portfolio choice.

\section{REFERENCES}

Brunnermeier, Markus K. 2001. Asset Pricing under Assymmetric Information. Oxford University Press.

Hassan, Tarek A., and Thomas M. Mertens. 2010. "The Social Cost of NearRational Investment." $N Y U$ Working Paper No. FIN-10-008.

Hassan, Tarek A., and Thomas M. Mertens. 2011. "Information Acquisition vs. Precision in Portfolio Choic." mimeo University of Chicago. 
Mertens, Thomas. 2009. "Excessively Volatile Stock Markets: Equilibrium Computation and Policy Analysis." mimeo New York University.

Niewerburgh, Stijn Van, and Laura Veldkamp. 2009. "Information Acquisition and Under-Diversification." mimeo NYU Stern. 